\title{
Managing Preterm Infants Born to COVID-19 Mothers: Evidence from a Retrospective Cohort Study in Wuhan, China
}

\author{
Xiaolin $\mathrm{Hu}^{\mathrm{a}}$ Jinzhi Gao ${ }^{\mathrm{a}}$ Ying Wei $^{\mathrm{a}}$ Hui Chen $^{\mathrm{b}}$ Xuan Sun ${ }^{\mathrm{a}}$ Juan Chen $^{\mathrm{c}}$ \\ Xiaoping Luo ${ }^{a}$ Ling Chen ${ }^{a}$ \\ a Department of Pediatrics, Tongji Hospital, Tongji Medical College, Huazhong University of Science and \\ Technology, Wuhan, China; ${ }^{b}$ Department of Obstetrics and Gynecology, Wuhan Union Hospital Xi Yuan, \\ Tongji Medical College, Huazhong University of Science and Technology, Wuhan, China; ' ${ }^{\circ}$ Department of \\ Biochemistry and Molecular Biology, Basic Medicine School, Tongji Medical College, Huazhong University of \\ Science and Technology, Wuhan, China
}

\section{Keywords}

Preterm infants COVID-19 mothers $\cdot$ Clinical outcomes

\begin{abstract}
Background: COVID-19 has spread rapidly over the world. Little is known about the outcomes of infections in pregnant women. The management and characteristics of preterm infants born to COVID-19 mothers need to be clarified. Methods: In this retrospective, single-center cohort study, we describe the clinical courses of 6 preterm infants born to COVID-19 mothers, the management protocol, and related outcomes. Results: Six preterm infants were admitted to Tongji Hospital between January 23 and March 19, 2020. Gestational age ranged from $28+5$ to $36+3$ weeks. One late preterm infant was delivered early due to maternal dyspnea from COVID-19. Five infants were delivered by Caesarean section. None had perinatal asphyxia. Two infants required respiratory support due to respiratory distress syndrome and apnea of prematurity. All infants did not develop severe complications of prematurity and are negative for severe acute respiratory syndrome (SARS)-CoV-2 nucleic acid testing. Conclusion: With an expedited and adequate delivery protocol, less invasive treatment principle, and active infection precautious, we found a limited impact of COVID-19
\end{abstract}

karger@karger.com www.karger.com/neo

Karger

GOPEN ACCESS
(C) 2020 S. Karger AG, Basel

Published by S. Karger AG, Basel

This is an Open Access article licensed under the Creative Commons Attribution-NonCommercial-4.0 International License (CC BY-NC) (http://www.karger.com/Services/OpenAccessLicense), applicable to the online version of the article only. Usage and distribution for commercial purposes requires written permission. mothers on preterm delivery and neonatal short-term outcomes. The risk of vertical transmission of SARS-CoV-2 is low in preterm infants born to COVID-19 mothers if appropriate management is implemented.

(C) 2020 The Author(s)

Published by S. Karger AG, Basel

\section{Introduction}

Since December 2019, a series of viral pneumonia called coronavirus disease 2019 (COVID-2019) has spread rapidly from Wuhan (China) to all over the world [1], and severe acute respiratory syndrome (SARS)-CoV-2 was found to be the pathogenic factor for this disease [2]. Confirmed cases were steeping up with an increasing number of pregnant women diagnosed. However, clinical data on pregnancy infection are rare $[3,4]$, and vertical transmission from mothers to infants is unknown [5]. Most research has described mothers and their term infants, while the management and characteristics of preterm infants born to COVID-19 mothers require to be clarified. In this study, we describe the clinical course of preterm infants born to mothers with COVID-19, management, and related outcomes. 


\section{Materials and Methods}

\section{Study Design and Participants}

We included preterm infants (gestational age $[\mathrm{GA}]<37$ weeks) born to mothers with COVID-19 between January 23 and March 19. The diagnosis of maternal COVID-19 was based on the positive result of SARS-CoV-2 nucleic acid testing or serologic tests according to the WHO guideline [6] and the guideline implemented by the National Health Commission of People's Republic of China [7]. This study was approved by the ethics committee of Tongji Hospital (approval No. TJ-C20200134). Written informed consents were obtained from patients before enrollment.

\section{Patient Management}

A multidisciplinary team, including obstetricians, infectious disease physicians, and neonatologists, was consulted prior to delivery. Timing and mode of delivery and delivery site were determined based on the maternal and fetal risks. Dexamethasone was given to mothers to promote fetal lung maturation as per standard recommendations. Caesarean section was chosen in a low threshold. Neonatal Resuscitation Program guidelines were followed; however, the umbilical cord was clamped immediately after birth, and mother-baby contact was discouraged to reduce the infection risk to infants.

All neonates born to COVID-19 mothers were isolated immediately after birth and transferred to the isolation center. They were quarantined in a single room initially and transferred to a common room after SARS-CoV-2 infection had been ruled out. The infants were monitored for vital signs, including oxygen saturation, and closely observed for signs of respiratory distress or sepsis. Respiratory support, such as continuous positive airway pressure and oxygen, was used to maintain oxygen saturation above $90 \%$. Caffeine was prescribed for infants $<32$ weeks' GA. Invasive procedures were avoided if possible; however, in case of the requirement of parenteral nutrition, a central line, such as a peripheral inserted central catheter (PICC), was inserted to prevent repeated peripheral puncture. Feeds (preterm formula) were started early and advanced as tolerated. Clinical examinations were avoided. Infants were isolated for 14 days as long as repeated nucleic acid test remained negative [8].

\section{Data Collection}

Clinical data were collected from both mothers and infants. Preterm infants during hospitalization were recorded for respiratory distress or infections and short-term complications related to premature birth, such as abnormal head ultrasound as intraventricular hemorrhage, gastrointestinal disturbance as feeding intolerance and signs of necrotizing enterocolitis (NEC), apnea of prematurity, bronchopulmonary dysplasia (BPD), retinopathy of prematurity, and prolonged jaundice or cholestasis. Laboratory tests and the relevant tests for SARS-CoV-2 infection were also recorded. Data were extracted primarily from the medical records by 2 investigators (X.H. and J.G.).

SARS-CoV-2 nucleic acids were tested by real-time polymerase chain reaction (RT-PCR) in Tongji Hospital (qualitative SARSCoV-2 RT-PCR, Guangzhou Daan Biotechnology Co., Ltd.), and details on the times and sites for RT-PCR are shown in Table 1. COVID-19 was diagnosed based on SARS-CoV-2 nucleic acid detection from the respiratory tract as previously reported [9]. All preterm infants were tested for the nucleic acid from throat and anal swabs. Amniotic fluid and umbilical cord blood at delivery, gastric aspiration before feeding, urine and stool samples, and mothers' expressed breast milk were also collected for testing for SARS-CoV-2 nucleic acid. SARS-CoV-2-specific serological tests were performed by chemiluminescent immunoassay (iFlash 3000 Chemiluminescence Immunoassay Analyzer, YHLO Biotech Co., Ltd., Shenzhen, China) in mothers at delivery and in infants' blood samples immediately, 7,14 , and 28 days after birth [10].

\section{Outcomes}

The primary outcome is whether the preterm infants had SARS-CoV-2 infection. The secondary outcomes are any shortterm complications, such as sepsis, head ultrasound abnormality, patent ductus arteriosus, BPD, NEC, prolonged jaundice caused by cholestasis, and retinopathy of prematurity. Head ultrasound abnormality included either grade 3-4 intraventricular hemorrhage or periventricular leukomalacia; BPD is defined as relay on oxygen for $>28$ days per NICHD guideline. NEC is specific to stages 2-3. Cholestasis is defined as when direct bilirubin is $>17.1 \mu \mathrm{mol} / \mathrm{L}$.

\section{Results}

\section{General Demographic and Obstetric Features}

Forty-two neonates delivered by COVID-19 mothers were admitted to our tertiary neonatal isolation centers. None of them were tested positive for SARS-CoV-2 infection. Six preterm infants, including one set of twins, were delivered from January 23 to March 19, 2020. They were isolated immediately after birth and admitted to our intensive neonatal isolation center for further assessment. None of the infants had congenital dysmorphic features. One infant was delivered in the obstetric department of our hospital, while the other 5 were transferred from referral hospitals. One was a girl and the other 5 were boys (Table 2). The age of mothers ranged from 26 to 36 years with a median age of 30.3 years. Only 1 mother had a previous heart issue, while the others were healthy. The GA of the infants ranged from 28 to $36+3$ weeks with an average GA of $33+3$ weeks (Table 2). Four infants were late preterm and 2 were premature infants. The reasons for preterm birth were maternal deterioration of pneumonia and shortness of breath in 1 patient, maternal heart issue in 1 patient, premature rupture of membranes with/without preterm labor in the 2 preterm infants, and severe preeclampsia in the $35+6$ weeks' GA twins. Five preterm infants were delivered by Caesarean section per the decision of the expert team, whereas 1 was born by inevitable spontaneous vaginal delivery at 28 weeks' GA. Dexamethasone was administrated prenatally in all 6 patients. None of the infants had asphyxia. Two mothers had elevated C-reactive protein at delivery. Normal placenta and clear amniotic fluid ruled out chorioamnionitis in all mothers. 


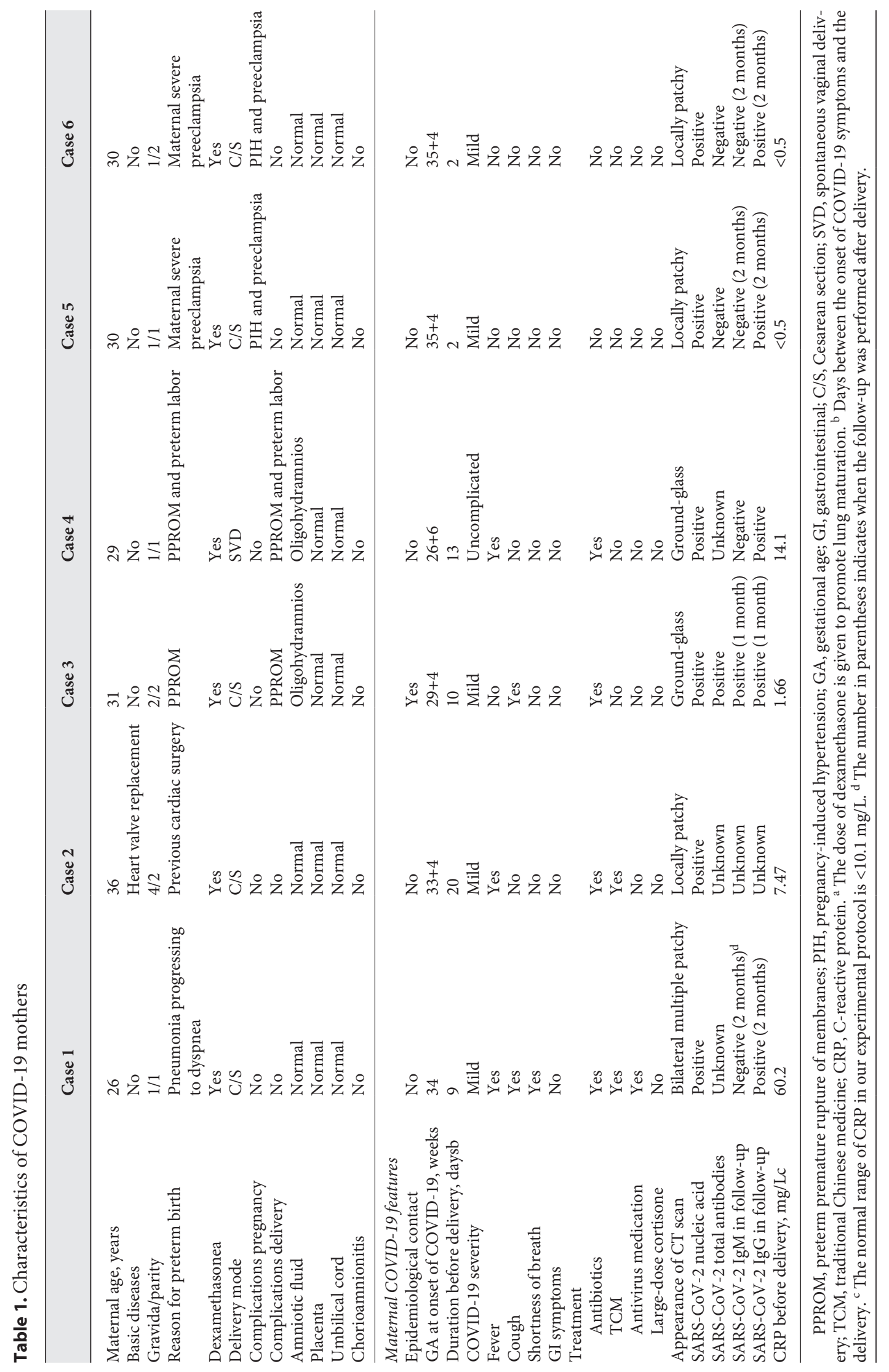


Table 2. Characteristics of preterm infants born to COVID-19 mothers

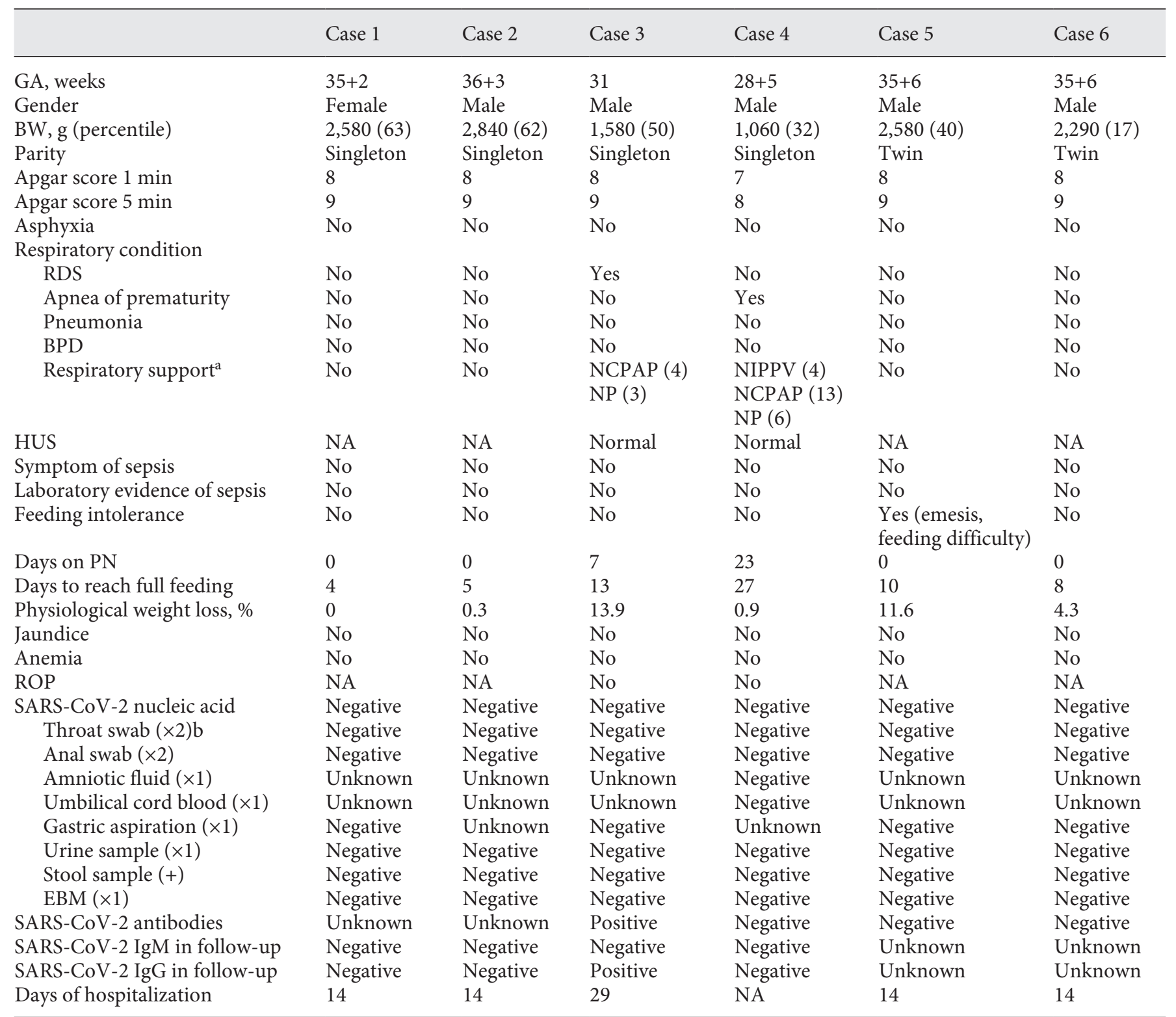

GA, gestational age; BW, birth weight; RDS, respiratory distress syndrome; BPD, bronchopulmonary dysplasia; HUS, head ultrasound; PN, parenteral nutrition; ROP, retinopathy of prematurity; NIPPV, noninvasive positive pressure ventilation; NCPAP, nasal continuous positive airway pressure; NP, nasal prone; NA, not available; EBM, expressed breast milk. ${ }^{\text {a }}$ The numbers in parentheses represent the number of days the infants received respiratory support. ${ }^{b}$ The numbers in parentheses indicate the times the samples were tested.

\section{Maternal Clinical Features of COVID-19}

As for the maternal COVID-19 clinical manifestations, only 1 mother had an obvious contact history since both her husband and brother had the infection. Most mothers did not deliver immediately after the infection. Days between the onset of COVID-19 and delivery ranged from 2 to 20 days with an average of 9 days. One mother was infected in the late second trimester $(26+6$ weeks), while the others were infected in the third trimester. Clinical presentations were either uncomplicated infections or mild pneumonia. The symptoms included fever in 3 mothers, progressive cough in 2 mothers, shortness of 
breath in 1 late preterm mother, and asymptomatic except for an abnormal chest CT scan. None of the mothers required oxygen therapy nor admittance to the intensive care unit. The manifestations of chest $\mathrm{CT}$ scans were not severe. Only 1 mother had progression to multiple bilateral consolidation, 2 had a local lesion on a unique lobe compatible with viral infection, and 2 had atypical ground glass appearance. The SARS-CoV-2 nucleic acid testing confirmed the infection in all pregnant women. Two mothers had SARS-CoV-2-specific serological test. One patient had positive SARS-CoV-2 IgG, and none had positive SARS-CoV-2-specific IgM 1 day before delivery. In the follow-up of 5 mothers, all had positive $\operatorname{IgG}$, while only 1 had positive IgM (Table 1). No mother was treated with anti-virus medication and large doses of cortical steroids in the cohort.

\section{Clinical Features and Short-Term Outcomes}

The clinical course during hospitalization was insignificant, and the short-term outcomes of the premature infants were mostly favorable. Birth weights were all adequate for GA, while none of the infants had perinatal asphyxia. Of the 4 late preterm infants, none had respiratory distress nor required respiratory support. Two premature infants required noninvasive ventilation for 7 and 23 days due to respiratory distress syndrome and apnea of prematurity, but neither developed BPD. Surfactants were not used. One twin born at $35+6$ weeks' GA had initial feeding intolerance present with recurrent regurgitation, abdomen distension, sucking weakness, and feeding difficulty. The twin's achievement of full feeding was delayed to 10 days of life due to this issue, and the physical weight loss reached $300 \mathrm{~g}$ without parental nutrition; the blood sugar was normal. Peripheral intravenous parenteral nutrition was discontinued on day of life 7 in the 31 weeks' GA infant, who reached full feeding on the 13th day after birth, while a PICC was inserted immediately after birth in the 28 weeks' GA preterm infant who was treated with parenteral nutrition for 23 days overall. Head ultrasound in 2 premature infants was normal without patent ductus arteriosus. Chest X-ray then ruled out pneumonia and parenchymal lung disease. Infection and sepsis were not observed during hospitalization in any of the preterm infants. None of the preterm infants developed gastrointestinal complications, such as NEC and severe cholestasis, and none had severe organ dysfunctions. Retinopathy of prematurity has been ruled out so far, but follow-up is necessary. All late preterm infants met discharging criteria at the end of isolation. The 31 weeks' GA infant was discharged on day 29 of life with a weight of
$2,020 \mathrm{~g}$. The 28 weeks' GA infant was still in the hospital for feeding and growth. SARS-CoV-2 nucleic acid testing was negative in all infants from samples of throat and anal swabs. Amniotic fluid and umbilical cord blood (in 1 infant) at delivery, gastric aspiration before feeding (in 4 infants), urine and stool samples (in 6 infants), and breast milk (5 mothers) also showed undetectable SARS-CoV-2 nucleic acid, while SARS-CoV-2-specific IgG was found in 1 premature infant (25\%) given that the test was only done in 4 infants. None of the babies had positive IgM, while we found that case 3 had positive IgG (Table 2). The titer of SARS-CoV-2 IgG in this infant declined rapidly from 142.00 to $11.25 \mathrm{AU} / \mathrm{mL}$ in the following 75 days.

\section{Discussion/Conclusion}

The main target of SARS-CoV-2 virus is the human lung, though the kidney and liver may also be damaged [11]. Mechanisms of mortality and morbidity are unclear; however, multiorgan dysfunction caused by inflammatory cascade may be crucial. A placental biopsy found nonspecific inflammatory deposit and massive placenta infarction [12]. Therefore, the impact of maternal COVID-19 on the fetus and neonate is a big concern. On the one hand, maternal-fetal vertical transmission is controversial. Data from several infected infants with early onset recently indicated that neonates were less vulnerable to this transmission route [13]. Additionally, studies on neonates born to mothers infected with SARS-CoV-2 close to parturient stage ( $<1$ week) showed good clinical outcomes $[14,15]$; however, preterm infants were not always delivered immediately upon maternal diagnosis, and they were more susceptible to maternal inflammation and placenta disturbance, which was relevant to intrauterine growth retardation, fetal distress, and other complications [16]. Therefore, preterm infants born to COVID-19 mothers should be distinguished and assessed independently from term infants. In our cohort, SARS-CoV-2 infection was found in none of the preterm infants prenatally and postnatally. One infant had positive SARS-CoV-2-specific IgG after birth, which was possibly transplacental passively from the mother since it declined rapidly.

It has been reported previously that SARS was correlated with spontaneous abortion, preterm birth, and small for GA infants [17]. In addition, Middle East Respiratory Syndrome (MERS) was reported to be associated with birth, prematurity, and infant death [18]. From January to March 2020, 42 infants born to COVID-19 mothers were admitted to our center, while 6 were preterm infants. 
The risk of preterm birth in our cohort (14.3\%) is in the range of the ordinary population [19] but lower than those in SARS and MERS mothers. In addition, the risk of preterm birth has been shown to be lower than in SARS and MERS mothers as well as in Pandemic (H1N1) 2009 mothers [20]. In most practices in Wuhan, pregnancies close to term ( $>35$ weeks) may be considered for termination, while earlier delivery is prevented if the disease permits it; the time for delivery was determined in a case-tocase pattern [21]. In our research, we found that only 1 late preterm birth was related to COVID-19 since the mother had shortness of breath affecting the pregnancy. The other preterm births were mainly due to either maternal basic disease or obstetric complications, and times of deliveries were far behind the infection. We found no predominant evidence of infants' SARS-CoV-2 infection, although the decision for delivery was based on obstetric status in an expedited schedule pattern rather than COVID-19 pneumonia itself.

Infants born to mothers with COVID-19 should be considered as suspected patients to be under isolation and surveillance [22]. For pregnant women expecting premature delivery, treatments for COVID-19 are mainly conservative. Antivirus medication and large doses of cortical steroids are mostly not used since there is no confirmed effect and they may lead to unfavorable consequences in preterm infants [23-25]. Temperature stabilization and respiratory support is always critical in managing the preterm newborn in the delivery room, and personal protective equipment is adequately applied. The principle of preventing unnecessary invasive procedures is implemented in our center; however, we did insert a PICC line to a 28 weeks' GA preterm infant immediately after birth when anticipating its necessity. No SARS-CoV-2 has been found yet in the expressed breast milk of delivered mothers; however, expressed breast milk was not used in preterm infants in our isolation center. Although transmission directly from the breast milk is unlikely, the processing procedure, such as milk expression and containing, could not be guaranteed. By performing the above, we assured an optimal preterm outcome, while at the same time preventing any nosocomial infection.

One limitation of our study is the sample size. Extremely preterm infants were not included in our study (the lowest GA in our study is $28+5$ weeks with a birth weiht of $1,060 \mathrm{~g})$. Additionally, we only monitored infants for a short period. Long-term follow-up is needed. Finally, samples from the placenta, amniotic fluid, and umbilical cord were not assessed and investigated in infants transferred from referral hospitals.
In conclusion, by utilizing a comprehensive approach and multidisciplinary attendance, the clinical courses of the preterm infants were favorable, and the short-term outcomes were optimal. No cases of vertical transmission were found in our cohort. We provide the first evidence on the practice approaches when managing preterm infants with maternal COVID-19 in the intensive neonatal isolation unit.

\section{Acknowledgement}

We thank the staff in our Tertiary Neonatal Isolation Center (Neonatal Department, Pediatric Department, Tongji Hospital of Tongji Medical College, Huazhong University of Science and Technology) for their hard work.

\section{Statement of Ethics}

The research was conducted ethically in accordance with the World Medical Association Declaration of Helsinki. This study was approved by the Ethics Committee of Tongji Hospital, Tongji Medical College, Huazhong University of Science and Technology. Written informed consents were obtained from the patients before the enrollment.

\section{Conflict of Interest Statement}

The authors have no conflicts of interest to declare.

\section{Funding Sources}

This work was supported by Hubei Emergency Response Fund (2020FCA006) and Hubei Provincial Science Foundation (WJ2019M125).

\section{Author Contributions}

All authors had full access to all the data in the study and take responsibility for the integrity of the data and the accuracy of the data analysis. L.C. and X.L. were responsible for the study concept and design; X.H. and J.G. were responsible for the acquisition, analysis, and interpretation of the data and drafting of the manuscript; Y.W. and H.C. were responsible for data collection; X.S. was responsible for analysis of the data; J.C. was responsible for the revision of the manuscript. 


\section{References}

1 Huang C, Wang Y, Li X, Ren L, Zhao J, Hu Y, et al. Clinical features of patients infected with 2019 novel coronavirus in Wuhan, China. Lancet. 2020 Feb;395(10223):497-506.

2 Wu F, Zhao S, Yu B, Chen YM, Wang W, Song ZG, et al. A new coronavirus associated with human respiratory disease in China. $\mathrm{Na}$ ture. 2020 Mar;579(7798):265-9.

3 Chen H, Guo J, Wang C, Luo F, Yu X, Zhang $\mathrm{W}$, et al. Clinical characteristics and intrauterine vertical transmission potential of COVID-19 infection in nine pregnant women: a retrospective review of medical records. Lancet. 2020 Mar;395(10226):809-15.

4 Schwartz DA. An Analysis of 38 Pregnant Women with COVID-19, Their Newborn Infants, and Maternal-Fetal Transmission of SARS-CoV-2: Maternal Coronavirus Infections and Pregnancy Outcomes. Arch Pathol Lab Med. 2020.

5 Shek CC, Ng PC, Fung GP, Cheng FW, Chan $\mathrm{PK}$, Peiris MJ, et al. Infants born to mothers with severe acute respiratory syndrome. Pediatrics. 2003 Oct;112(4):e254.

6 World Health Organization. Clinical management of severe acute respiratory infection when novel coronavirus ( $\mathrm{nCoV}$ ) infection is suspected: interim guidance. WHO; 2020.

7 Released by National Health Commission \& National Administration of Traditional Chinese Medicine on March 3, 2020. Diagnosis and Treatment Protocol for Novel Coronavirus Pneumonia (Trial Version 7). Chin Med J (Engl). 2020 May;133(9):1087-95.

8 Wang L, Shi Y, Xiao T, Fu J, Feng X, Mu D, et al.; Working Committee on Perinatal and Neonatal Management for the Prevention and Control of the 2019 Novel Coronavirus Infection. Chinese expert consensus on the perinatal and neonatal management for the prevention and control of the 2019 novel coronavirus infection (First edition). Ann Transl Med. 2020 Feb;8(3):47.
9 Liu W, Zhang Q, Chen J, Xiang R, Song H, Shu S, et al. Detection of Covid-19 in Children in Early January 2020 in Wuhan, China. N Engl J Med. 2020 Apr;382(14):1370-1.

$10 \mathrm{Xu} \mathrm{W}$, Li J, He X, Zhang C, Mei S, Li C, et al. The diagnostic value of joint detection of serum IgM and IgG antibodies to $2019-\mathrm{nCoV}$ in 2019-nCoV infection. Chin J Lab Med. 2020; 43.

11 Diao B, Feng Z, Wang C, Wang H, Liu L, Wang $C$, et al. Human Kidney is a Target for Novel Severe Acute Respiratory Syndrome Coronavirus 2 (SARS-CoV-2) Infection. medRxiv. 2020

12 Chen S, Huang B, Luo DJ, Li X, Yang F, Zhao $\mathrm{Y}$, et al. [Pregnant women with new coronavirus infection: a clinical characteristics and placental pathological analysis of three cases]. Zhonghua Bing Li Xue Za Zhi. 2020 May 8; 49(5):418-23.

13 Zeng L, Xia S, Yuan W, Yan K, Xiao F, Shao J, et al. Neonatal Early-Onset Infection With SARS-CoV-2 in 33 Neonates Born to Mothers With COVID-19 in Wuhan, China. JAMA Pediatr. 2020 Mar 26;e200878

14 Yu N, Li W, Kang Q, Xiong Z, Wang S, Lin X, et al. Clinical features and obstetric and neonatal outcomes of pregnant patients with $\mathrm{CO}$ VID-19 in Wuhan, China: a retrospective, single-centre, descriptive study. Lancet Infect Dis. 2020 May;20(5):559-64.

15 Wang WL, Wang HJ, Deng Y, Song TE, Lan JM, Wu GZ, et al. Characterization of antiMERS-CoV antibodies against various recombinant structural antigens of MERS-CoV in an imported case in China. Emerg Microbes Infect. 2016 Nov;5(11):e113.
16 Kalagiri RR, Carder T, Choudhury S, Vora N Ballard AR, Govande V, et al. Inflammation in Complicated Pregnancy and Its Outcome. Am J Perinatol. 2016 Dec;33(14):1337-56.

17 Wong SF, Chow KM, Leung TN, Ng WF, Ng TK, Shek CC, et al. Pregnancy and perinatal outcomes of women with severe acute respiratory syndrome. Am J Obstet Gynecol. 2004 Jul;191(1):292-7.

18 Alserehi H, Wali G, Alshukairi A, Alraddadi B. Impact of Middle East Respiratory Syndrome coronavirus (MERS-CoV) on pregnancy and perinatal outcome. BMC Infect Dis. 2016 Mar;16(1):105.

19 Vogel JP, Chawanpaiboon S, Moller AB, Watananirun $\mathrm{K}$, Bonet $\mathrm{M}$, Lumbiganon $\mathrm{P}$. The global epidemiology of preterm birth. Best Pract Res Clin Obstet Gynaecol. 2018 Oct:52:3-12

20 Takahashi N, Kitajima H, Kusuda S, Morioka I, Itabashi K. Pandemic (H1N1) 2009 in neonates, Japan. Emerg Infect Dis. 2011 Sep; 17(9):1763-5.

21 Qi H, Luo X, Zheng Y, Zhang H, Li J, Zou L, et al. Safe Delivery for Pregnancies Affected by COVID-19. BJOG. 2020 Jul;127(8):927-9.

22 [Recommendations for the diagnosis, prevention and control of the 2019 novel coronavirus infection in children (first interim edition)]. Zhonghua Er Ke Za Zhi. 2020 Mar; 58(3):169-74.

23 Tegethoff M, Pryce C, Meinlschmidt G. Effects of intrauterine exposure to synthetic glucocorticoids on fetal, newborn, and infant hypothalamic-pituitary-adrenal axis function in humans: a systematic review. Endocr Rev. 2009 Dec;30(7):753-89.

24 Kochhar DM, Penner JD, Knudsen TB. Embryotoxic, teratogenic, and metabolic effects of ribavirin in mice. Toxicol Appl Pharmacol. 1980 Jan;52(1):99-112.

25 Ferm VH, Willhite C, Kilham L. Teratogenic effects of ribavirin on hamster and rat embryos. Teratology. 1978 Feb;17(1):93-101. 